\title{
Aktuelle Aspekte zu Diagnostik und Therapie der Osteoporose - Bedeutung für die Vermeidung von Frakturen bei postmenopausalen Frauen
}

\author{
Marius E. Kraenzlin ${ }^{1}$ und Christian Meier² \\ ${ }^{1}$ Endokrinologische Praxis \& Labor, CH-Basel; ${ }^{2}$ Klinik für Endokrinologie, Diabetologie und klinische Ernährung Universitätspital Basel
}

D ie Osteoporose ist eine systemische Skeletterkrankung, bei der eine Verschlechterung der Knochenarchitektur und der biomechanischen Eigenschaften sowie ein Verlust an Knochenmasse zu einer geringeren Knochenfestigkeit und damit $\mathrm{zu}$ einer erhöhten Knochenbrüchigkeit führt. Die klinische Bedeutung der Osteoporose ergibt sich aus ihren Komplikationen, den Frakturen, die nach einem inadäquaten Trauma auftreten. Die am meisten betroffenen Skelettregionen für osteoporotische Frakturen sind die Wirbelsäule, der proximale Femur und der distale Radius. Die durchschnittliche Wahrscheinlichkeit, im Laufe des Lebens eine osteoporotische Fraktur zu erleiden, beträgt im Alter von 50 Jahren für die Frau ca. 40-50\% und für den Mann ca. 20-30\%. In Abhängigkeit vom Alter beträgt bei postmenopausalen Frauen die Inzidenz für Wirbelfrakturen 5.8-29/1000/Jahr und für nicht-vertebrale Frakturen 19/1000/ Jahr [1, 2]. Osteoporotische Frakturen sind mit einer signifikanten Einschränkung der Lebensqualität und Alltagsfähigkeit als auch mit einer erhöhten Sterblichkeit vergesellschaftet [3-6]. In Anbetracht dieser Tatsachen stellt die Osteoporose nicht nur ein rasant wachsendes menschliches und medizinisches Problem dar, sondern auch eine zunehmende sozioökonomische Belastung unserer Gesellschaft.

Die aktuelle Knochenfestigkeit und Knochenmasse wird durch zwei Einflussgrössen bestimmt: einerseits durch den Knochenaufbau während der Adoleszenz und dem frühen Erwachsenenalter; andererseits durch das Ausmass des sich anschliessenden, altersbeding-

Hintergrund: Die klinische Bedeutung der Osteoporose ergibt sich aus ihren Komplikationen, den osteoporotischen Frakturen, davon ist jede 2. bis 3. postmenopausale Frau im Laufe ihres Lebens betroffen. Zielsetzung: Erstellung einer Übersicht zu diagnostischen und therapeutischen Möglichkeiten, wie sie heute in der Schweiz bei Osteoporose eingesetzt werden. Methoden: Systematische Selektion und Analyse der wissenschaftlichen Literatur zur Diagnostik und Therapie der Osteoporose, hauptsächlich in MEDLINE aus den Jahren 2000-2005. Ergebnisse und Schlussfolgerungen: Die Indikation zur Behandlung der Osteoporose stützt sich auf das individuelle Frak turrisiko, das durch die integrale Beurteilung verschiedener Risikofaktoren ermittelt wird. Die Kombination einzelner Risikofaktoren zur Voraussage des Frakturrisikos ist der alleinigen Bestimmung der Knochendichte überlegen. Die Entscheidung, welcher Art die Behandlung (präventiv, therapeutisch) sein soll, ergibt sich erst im Kontext aller Faktoren, die zu einer Erhöhung des Frakturrisikos führen und ist individuell zu fällen. Bei der Interpretation der Densitometrieresultate muss unterschieden werden zwischen der diagnostischen Schwelle und der Interventionsschwelle. Für verschiedene medikamentöse Wirkprinzipien (Bisphosphonate, Raloxifen, Teriparatid) ist eine signifikante Reduktion der Inzidenz vertebraler Frakturen dokumentiert. Eine Reduktion nicht-vertebraler Frakturen wurde insbesondere für die Bisphosphonate Alendronat und Risedronat als auch für Teriparatid gezeigt. Im klinischen Alltag richtet sich die Wahl eines spezifischen Therapeutikums nach dem individuellem Frakturrisiko und sollte unter Abwägung skeletaler und nichtskeletaler Medikamentenwirkungen bzw. -nebenwirkungen erfolgen.

Schlüsselwörter: Osteoporose, Diagnostik, Therapie

\section{Current Aspects of Diagnostics and Therapy of Osteoporosis:} Their Relevance for the Avoidance of Fractures in Postmenopausal Women

Background: Osteoporosis is of considerable clinical relevance due to the complications it causes i.e. osteoporotic fractures, which affect every second or third postmenopausal woman in the course of her life. Objective: To review the diagnostic and therapeutic standards of care as currently used in Switzerland in osteoporosis. Methods: Systematic selection and analysis of the relevant scientific literature on diagnostics and therapy of osteoporosis, primarily in MEDLINE from 2000-2005. Results and conclusions: The indication for the treatment of osteoporosis is based on individual fracture risk, as determined by the integral evaluation of different risk factors. The combination of different risk factors is a better basis for the prediction of fracture risk than is the determination of bone density alone. The decision as to the type of treatment (preventive, therapeutic) is taken individually, in the context of all factors which can increase the fracture risk. When interpreting the densitometry results, a distinction must be made between the diagnostic threshold and the threshold for intervention. A significant reduction in the incidence of vertebral fractures has been documented for several different drugs (bisphosphonates, raloxifene, teriparatide) Reduction in non-vertebral fractures has been demonstrated for the bisphosphonates alendronate and risedronate and also for teriparatide. In normal clinical practice, drugs are selected on the basis of individual fracture risk and should be based on consideration of the relative risk of skeletal and non-skeletal drug effects and on possible side effects.

Key words: Osteoporosis, diagnostics, therapy

ten Knochenmassenverlustes. Die maximale Knochenmasse, die in der Regel gegen Ende des zweiten Lebensjahrzehntes erreicht wird, wird durch eine Vielzahl von Faktoren wie Erbfaktoren, Hormonstatus, Ernährung und körperliche Aktivität bestimmt. Die hauptsächlichen Ursachen für den
Knochensubstanzverlust in der zweiten Lebenshälfte ist der nach der Menopause einsetzende Östrogenmangel bei der Frau, Alter, Lebensstil, Mangel- und Fehlernährung, Umweltfaktoren, sowie Krankheiten, die $\mathrm{zu}$ einem vermehrten Knochensubstanzverlust führen [7-10]. 


\section{Diagnostik}

Im frühen Stadium verursacht die Osteoporose keine Symptome. Die Abschätzung des Osteoporoserisikos ohne bereits eingetretene Fraktur ist daher schwierig, zumal bislang keine ausreichend sensitive und spezifische Untersuchungsmethode zur Früherkennung einer Osteoporose zur Verfügung steht. Damit wird heute eine „Case-Finding“ Strategie, die auf Erfassung von Hochrisiko-Personen ausgerichtet ist empfohlen [7, 11, 12]. Eine Indikation zur weiteren Abklärung ist bei erhöhtem Frakturrisiko (klinische Risikofaktoren) oder bei bereits erlittener Fraktur nach inadäquatem Trauma gegeben (Tabelle 1). Bei Vorliegen einer oder mehrerer der folgenden wichtigen Risikofaktoren sollte in jedem Fall eine weiterführende Diagnostik mittels Densitometrie erwogen werden: vorbestehende Frakturen nach inadäquatem Trauma, Langzeittherapie mit Glukokortikoiden, chronisch geringes Körpergewicht $\left(\mathrm{BMI}<20 \mathrm{~kg} / \mathrm{cm}^{2}\right)$, erhöhtes Sturzrisiko sowie Krankheiten, die mit einem erhöhten Frakturrisiko einhergehen.

\section{Densitometrie}

Die Bestimmung des Mineralgehaltes des Knochens (Osteodensitometrie) ist eine zuverlässige und weltweit gut validierte Methode zur Abschätzung des individuellen Frakturrisikos. Prospektive Untersuchungen haben gezeigt, dass das individuelle Frakturrisiko mit abnehmender Knochendichte exponentiell ansteigt $[7 ; 11 ; 13 ; 14]$. Als Faustregel gilt, dass eine Abnahme des Knochenmineralgehaltes an der Lendenwirbelsäule (L2-4) oder am Schenkelhals um eine Standardabweichung mit einem ca. 2-fach erhöhten Frakturrisiko am Messort verbunden ist (relatives Risiko=2). Die Bedeutung des Knochenmineralgehalts für das absolute Frakturrisiko wird allerdings stark vom Alter des Patienten beeinflusst: je höher das Alter, desto steiler der Gradient zwischen Knochendichte und Frakturwahrscheinlichkeit. Ein T-Wert von -2 SD ist demnach bei einem 80-jährigen Patienten mit einer wesentlich höheren absoluten Frakturwahrscheinlichkeit verbunden als bei einer jüngeren Person.

Tab. 1. Relative Risikofaktoren

\begin{tabular}{|c|c|}
\hline Hohes relatives Frakturrisiko $(\mathrm{RR} \geq 2)$ & Moderates relatives Frakturrisiko (RR 1-2) \\
\hline Alter $>70$ Jahre & $\begin{array}{l}\text { Chronischer Östrogen-Mangel, } \\
\text { Endogene Östrogenexpositionszeit < } 30 \text { Jahre }\end{array}$ \\
\hline Frühzeitige Menopause ( < 45 Jahre) & Calcium-Zufuhr < 300 mg/Tag \\
\hline $\begin{array}{l}\text { Hypogonadismus (beim Mann) oder } \\
\text { vorzeitige Menopause (<40 Jahre) }\end{array}$ & Primärer Hyperparathyreoidismus \\
\hline Bereits erlittene osteoporotische Fraktur & Therapie mit Antiepileptika \\
\hline $\begin{array}{l}\text { Schenkelhalsfraktur bei Verwandten } \\
\text { 1.Grades }\end{array}$ & Rheumatoide Arthritis \\
\hline Kortikosteroid-Therapie (früher, aktuell) & Morbus Bechterew \\
\hline Anorexia nervosa & Rauchen \\
\hline $\mathrm{BMI}<20 \mathrm{~kg} / \mathrm{m}^{2}$ & Alkoholabusus \\
\hline Ausgeprägte körperliche Inaktivität & Diabetes mellitus \\
\hline Chronische Niereninsuffizienz & Unbehandelte Hyperthyreose \\
\hline Organtransplantation & \\
\hline
\end{tabular}

Tab. 2. Densitometrische Klassifiktion der Osteoporose

Normal:

Knochendichtewerte (BMD) innerhalb 1 Standardabweichung (SD) vom Mittelwert junger Erwachsener (T-ScoreDensitometrische Klassifiktion der Osteoporose $\geq-1.0$ ).

Osteopenie (niedrige Knochenmasse):

Knochendichtewerte (BMD) mehr als 1 SD unter dem Mittelwert junger Erwachsener, aber weniger als 2.5 SD unterhalb dieses Wertes (T-Score -1.0 bis -2.5 ).

\section{Osteoporose:}

Knochendichtewerte (BMD) 2.5 SD oder mehr unterhalb des Mittelwertes für junge Erwachsene (T-Score $\leq-2.5$ ).

Schwere Osteoporose (etablierte Osteoporose):

Knochendichtewerte (BMD) 2.5 SD oder mehr unterhalb des Mittelwertes junger Erwachsener und Vorhandensein von einer oder mehreren Osteoporose-bedingten Frakturen.

Die WHO hat auf der Basis epidemiologischer Daten die Osteoporose der Frau nach der gemessenen Knochendichte definiert (Tabelle 2). Diese Definition der Osteoporose mittels T-Score gilt jedoch nur für die DXA-Methode und auch nur für postmenopausale Frauen; sie kann nicht auf andere Messmethoden (z.B. QCT oder Ultraschall) übertragen werden $[15,16)$. Im klinischen Gebrauch sollte die Knochendichtemessung als kontinuierlicher Risikofaktor in Ergänzung zu klinischen Risikofaktoren interpretiert werden. Während eine niedrige Knochendichte ein relativ gesteigertes Frakturrisiko anzeigt, ergibt sich aus der Knochendichtemessung allein kein Schwellenwert, der als Indikation zur
Behandlung beim individuellen Patienten herangezogen werden könnte.

\section{Quantitative \\ Ultraschalluntersuchungen}

Prospektive Untersuchungen zeigen, dass sich mit einer quantitativen Ultraschalluntersuchung (QUS) bei über 65jährigen Frauen, ähnlich wie mit der Densitometrie, das Frakturrisiko beurteilen lässt [17, 18]. Die Erniedrigung der Ultraschallmessgrösse am Kalkaneus um eine Standardabweichung ist mit einer Zunahme des relativen Risikos einer Hüftfraktur um den Faktor 1.7-2.5 assoziiert [18-20]. Allerdings muss bei der Anwendung der QUS berücksichtigt werden, dass die vorliegenden prospektiven Studien mit eini- 
gen ausgewählten Geräten durchgeführt wurden und daher die Ergebnisse dieser Studien nicht ohne weiteres auf andere QUS-Geräte übertragen werden können [21]. Zur Diagnose der Osteoporose kann die QUS nicht eingesetzt werden, da die WHO-Kriterien (T-Score) für diese Messmethode keine Gültigkeit haben. Ein nicht-normaler Wert der QUS kann jedoch als Ausgangspunkt für eine weitere Abklärung mittels DEXA gelten, vor allem in Gebieten, in denen der Zugang zu DXA-Geräten limitiert ist. Wegen einer relativ geringen Genauigkeit und Reproduzierbarkeit kann die Ultraschalluntersuchung des Knochens nicht zur Verlaufskontrolle empfohlen werden.

\section{Biochemische Marker des Knochenstoffwechsels:}

Ein erhöhter Knochenumbau wird als unabhängiger Risikofaktor für osteoporotische Frakturen und auch als prädiktiver Faktor für das Ansprechen auf eine antiresorptive Behandlung angesehen [22-24]. In mehreren prospektiven Studien konnte ein Zusammenhang zwischen dem Knochenumbau und dem Risiko einer osteoporotischen Fraktur (Wirbel und Schenkelhals) nachgewiesen werden, indem bei Frauen mit erniedrigtem Knochenmineralgehalt und erhöhter Knochenabbaurate das Frakturrisiko doppelt so hoch ausfiel wie bei Frauen mit erniedrigtem Knochenmineralgehalt und normaler Knochenabbaurate [23, 24]. Diese Untersuchungen weisen darauf hin, dass der erhöhte Knochenumbau, v.a. die mit den spezifischen Knochenumbaumarkern evaluierte Knochenabbaurate, eine unabhängige Determinante für das Frakturrisiko darstellt und damit eine bessere Beurteilung des Frakturrisikos erlaubt als die Knochendichte allein [23, 24].

\section{Evaluation des Frakturrisikos}

Zusammen mit dem Nachweis osteoporotischer Frakturen ist die Knochendichtemessung nach wie vor der Eckstein der Osteoporosediagnostik. Bei der Interpretation der Densitometrieresultate muss aber unterschieden werden zwischen der diagnostischen Schwelle (WHO-Definition der Osteoporose: T-Score -2.5 SD) und der
Interventionsschwelle. Ein T-Score von -2.5 SD gemessen am proximalen Femur bei einer 55-jährigen Frau ohne zusätzliche Risikofaktoren ist mit einer 10-Jahreswahrscheinlichkeit für eine osteoporotische Fraktur von nur ca. $11 \%$ assoziiert. Mit zunehmendem Alter steigt die Frakturwahrscheinlichkeit bei identischem T-Score jedoch an: mit 65 Jahren beträgt die Wahrscheinlichkeit ca. $20 \%$ und mit 70 Jahren sogar 25\% [14, 25, 26]. Das Lebensalter stellt damit bei Frauen und Männern einen wichtigen und von anderen Faktoren unabhängigen Risikofaktor für osteoporotische Frakturen dar. Neben Alter und Knochenmineralgehalt leisten auch prävalente Wirbelfrakturen einen unabhängigen Beitrag zum Frakturrisiko [14, 27-30]. Bei einer einzelnen Fraktur besteht ein 3-fach, bei 2 prävalenten Frakturen ein 10-fach und bei mehr als 3 Wirbelfrakturen ein über 20-fach erhöhtes Risiko einer erneuten Wirbelfraktur [31]. In mehreren Studien konnte ein Zusammenhang zwischen dem Knochenumbau und dem Risiko einer osteoporotischen Fraktur nachgewiesen werden: bei Frauen mit erniedrigtem Knochenmineralgehalt und erhöhter Knochenabbaurate (Knochenumbaumarker in Urin und Serum) fiel das Frakturrisiko doppelt so hoch aus wie bei Frauen mit erniedrigtem Knochenmineralgehalt und normaler Knochenabbaurate [23]. Ein niedriges Körpergewicht (d.h. BMI $<20 \mathrm{~kg} / \mathrm{m}^{2}$ ) ist mit einem 2-fach erhöhten Risiko einer Schenkelhalsfraktur assoziiert [14]. Stürze in der Anamnese gehen mit einem erhöhten Risiko, vor allem für periphere Frakturen und Schenkelhalsfrakturen, aber auch Wirbelfrakturen, einher. In Kürze wird ein Algorhythmus - basierend auf den oben erwähnten Faktoren - vorgestellt, welcher erlauben wird, die individuelle 10-Jahres-Frakturwarscheinlichkeit zu berechnen.

\section{Therapie}

Die klinische Bedeutung der Osteoporose wird durch das Auftreten nichttraumatischer Frakturen bestimmt. Entsprechend ist es das vordergründige
Ziel, bei betroffenen Frauen die Frakturinzidenz zu reduzieren. In den letzten 10 Jahren sind mehrere grosse placebokontrollierte Doppelblindstudien mit dem klinischen Endpunkt neuer vertebraler und nicht-vertebraler Frakturen bei postmenopausalen Frauen publiziert worden. Dabei konnte für verschiedene medikamentöse Wirkprinzipien (Bisphosphonate, Raloxifen, Teriparatide) eine signifikante Reduktion der Inzidenz vertebraler Frakturen zwischen 30 und $65 \%$ dokumentiert werden. Eine Reduktion nicht-vertebraler Frakturen wurde insbesondere für Alendronat und Risedronat wie auch für Teriparatid gezeigt. Im klinischen Alltag richtet sich die Wahl eines spezifischen Therapeutikums nach dem individuellem Frakturrisiko und sollte unter Abwägung skeletaler und nicht-skeletaler Medikamentenwirkungen bzw. -nebenwirkungen erfolgen.

\section{Kalzium und Vitamin D}

Eine ausreichende Kalziumzufuhr beeinflusst wesentlich den Aufbau und Erhalt des Knochenmineralgehaltes. Eine Supplementation von Kalzium und Vitamin $\mathrm{D}_{3}$ stellt daher eine Basisintervention in der Prävention und $\mathrm{Be}$ handlung der postmenopausalen Osteoporose dar. Die empfohlenen Tagesdosen von Kalzium liegen für postmenopausale Frauen zwischen 1000 und 1500 mg mit einer höheren Richtdosis für postmenopausale Frauen ohne Hormonersatzbehandlung $\quad[11,32]$. Für Vitamin $\mathrm{D}_{3}$ wird eine tägliche $\mathrm{Zu}$ fuhr von 800 IE empfohlen.

Die Wirksamkeit einer kombinierten Kalzium und Vitamin D Supplementation in der Reduktion nicht-vertebraler Frakturen konnte in drei grossen placebokontrollierten Primärpräventionstudien bei Altenheimbewohnerinnen [33] und nicht-institutionalisierten Frauen [34] dokumentiert werden. Vor allem die Arbeiten bei Altenheimbewohnerinnen waren darin gekennzeichnet, dass bei vielen der älteren Studienteilnehmerinnen ein Kalzium- und Vitamin-D-Mangel vor Studieneintritt vorgelegen hat. Durch eine Supplementation mit $1200 \mathrm{mg}$ Kalzium und 800 IE Vitamin D über 18 Monate konnte bei 3270 Frauen (mittleres Alter 84 Jahre) die Inzidenz 
von Schenkelhalsfrakturen um 43\% und die Inzidenz nicht-vertebraler Frakturen um $32 \%$ gesenkt werden [33].

In kürzlich publizierten Sekundärpräventionsstudie bei Männern und Frauen mit osteoporotischen Frakturen konnte weder mit Kalzium alleine, Vitamin D alleine, noch mit einer Kombinationsbehandlung gegenüber Placebo das Auftreten neuer Frakturen verhindert werden [35]. Ingesamt genügt aber eine Kalzium- und VitaminD-Supplementation in der Regel nicht, um das Frakturrisiko in einem genügenden Mass zu senken, vor allem bei jüngeren Patienten. Dies wurde kürzlich auch mit den Resultaten der WHIStudie gezeigt [35A, 35B]. In dieser Studie wurde bei einer Kalzium-Einnahme von durchschnittlich $2000 \mathrm{mg}$ täglich auch ein vermehrtes Auftreten von Urolithiasis beobachtet. Eine genügende Versorgung mit Calcium (1200-1500 mg täglich für postmenopausale Frauen) und Vitamin D stellt die Basis, einerseits für die Prävention wie auch für die Behandlung der Osteoporose, dar.

\section{Hormonersatztherapie}

Die Östrogendefizienz ist eine der Hauptrisikofaktoren in der Pathogenese der postmenopausalen Osteoporose. Bis zum Erscheinen der „Woman's Health Initiative, (WHI) basierte die Annahme, dass eine Hormonersatzbehandlung $\mathrm{zu}$ einer signifikanten Reduktion der Frakturrate bei Osteoporose führt, vor allem auf Kohortenstudien und zwei kleineren randomisierten kontrollierten Studien.

Erst vor kurzem sind zwei grosse prospektive Studien erschienen, die den Effekt einer Hormonsubstitutionsbehandlung auf das Risiko von koronaren Ereignissen in der Sekundärprävention (HERS - Heart and Estrogen/progestin Replacement Study) [36] und auf Nutzen und Risiken einer Hormontherapie in der Primärprävention (WHI - Women's Health Initiative) [37] untersucht haben. Bei keiner dieser Studien war das Vorliegen einer Osteoporose bzw. von Osteoporoserisikofaktoren ein Einschlusskriterium zur Studienteilnahme. In der HERSStudie wurde kein Unterschied in Bezug auf die Frakturinzidenz zwi- schen hormonbehandelten Frauen und Kontrollen beobachtet [36]. In der WHI-Studie dagegen, in der 16’608 postmenopausale Frauen über einen medianen Zeitraum von 5.2 Jahre beobachtet wurden, zeigte sich, dass konjugierte equine Östrogene und Medroxyprogesteronacetat zur Frakturreduktion geeignet sind. Während der gesamten Studiendauer traten 5 Schenkelhalsfrakturen weniger pro 10’000 hormonell behandelte Frauen/Jahr im Vergleich zur Placebogruppe auf. Eine ähnliche Frakturreduktion liess sich für vertebrale Frakturen dokumentieren [37]. Im kürzlich publiziertem Östrogenarm der WHI-Studie wurden vergleichbare Reduktionen des relativen Frakturrisikos sowohl für vertebrale als auch für nicht-vertebrale Frakturen beobachtet [38]. In der Gesamtbeurteilung der WHI-Studie, welche wegen eines erhöhten Risikos kardiovaskulärer Erkrankungen und einer Zunahme des Mammakarzinomrisikos vorzeitig abgebrochen wurde, kamen die Autoren jedoch zu Schluss, dass der Nutzen einer Hormontherapie nicht höher zu bewerten war als die potentiellen Risiken. Mit der neuen Datenlage ist es sicher so, dass man das Risiko-Nutzenverhältnis beim Einsatz einer Hormonersatzbehandlung, vor allem der kombinierten Ersatzbehandlung (Oestrogene und Gestagene), abwägen muss. Der Einsatz einer Hormonersatzbehandlung ist vor allem in den ersten Jahren der Menopause, wenn gleichzeitig auch klimakterische Beschwerden bestehen, zu sehen.

\section{Selektive Östrogen-Rezeptor Modulatoren (SERM)}

Raloxifen ist ein selektiver Östrogenrezeptor-Modulator (SERM) der zweiten Generation mit gewebespezifischen östrogen-agonistischen (Knochen, Leber) und östrogen-antagonistischen (Brust) Wirkungen. Bei Frauen mit Osteoporose reduziert Raloxifen das Risiko vertebraler Frakturen um ca. $30 \%$. Ein günstiger Effekt auf nichtvertebrale Frakturen konnte nur in einer post-hoc Analyse von Hochrisikopatienten gezeigt werden [39].

Als günstige extra-skeletale Wirkungen von Raloxifen sind eine Reduktion des Auftretens Östrogenrezeptorpositiver Mamakarzinome und eine mögliche Senkung der kardiovaskulären Morbidität bekannt. Der Einsatz von Raloxifen ist v.a. auf ältere postmenopausale Frauen beschränkt, da nicht selten bei jüngeren Frauen klimakterische Beschwerden ausgelöst werden können.

\section{Bisphosphonate}

Bisphosphonate machen ca. 70\% der Weltmarktes an Osteoporosemedikamenten aus. Ihre Hauptwirkung beruht auf einer Hemmung der osteoklastenvermittelten Knochenresorption. Die orale Bioverfügbarkeit von Bisphosphonaten beträgt nur ca. 1-3\% der eingenommenen Dosis und wird durch eine gleichzeitige Nahrungseinnahme zusätzlich beeinträchtigt. Durch die ausgeprägte Knochenaffinität wird nach der Einnahme ca. die Hälfte der Bisphosphonatdosis in den Knochen eingebaut, der Rest wird renal eliminiert. Die Halbwertszeit von Bisphosphonaten im Knochen kann mehrere Jahre betragen.

Studien mit Alendronat bei postmenopausalen Frauen mit vorbestehenden Wirbelkörperfrakturen zeigen, dass, verglichen zu Placebo, das Risiko neuer Frakturen (Wirbelkörper, Schenkelhals, distaler Radius) um ca. 50\% gesenkt werden kann [40]. Bei Frauen ohne Frakturanamnese, aber mit niedriger Knochendichte (T-Score $\leq 2.5 \mathrm{SD}$ ), konnte ebenfalls eine effektive Senkung des Frakturrisikos erzielt werden [41]. Die Wirkung von Alendronat auf die Knochendichte und den Knochenturnover nimmt nach dessen Absetzen verzögert ab. Kürzlich wurden Daten über den Langzeitverlauf der Knochendichte bis zu 10 Jahren publiziert [42], die längsten Untersuchungen mit dem klinischen Endpunkt osteoporotische Fraktur betragen 3 Jahre. Es ist jedoch nicht anzunehmen, dass der günstige Effekt auf die Frakturreduktion im Verlauf der Therapie abnimmt, ein Konsens über die optimale Therapiedauer liegt jedoch nicht vor.

Auch für Risedronat liegen mehrere Studien vor, die eine signifikante Reduktion des vertebralen und nichtvertebralen Frakturrisikos bei Frauen mit oder ohne vorbestehende Frakturen aufzeigen [43, 44]. Langzeitstudien 
mit dokumentierter Frakturreduktion liegen für Risedronat bis zu 5 Jahre vor. Beide Medikamente sind bezüglich ihres Nebenwirkungsprofils vergleichbar, für beide Substanzen liegen wöchentliche Applikationsformen vor, die in ihrer Wirkung auf die Knochendichte und den Knochenumbau mit den täglichen Tabletteneinnahmen vergleichbar sind. Direkte „head-to head“Vergleiche bezüglich ihrer Wirksamkeit in der Frakturprävention liegen nicht vor.

Ibandronat senkt bei postmenopausalen Frauen mit vorbestehenden Wirbelkörperbrüchen das Risiko neuer vertebraler Frakturen. Die Wirksamkeit auf nicht-vertebrale Frakturen wurde bis anhin nur in einer post-hoc Analyse von Hochrisikopatientinnen gezeigt [45].

\section{Parathormon}

Teriparatid ist ein biotechnologisch hergestelltes aktives Fragment des endogenen Parathormons (rhPTH [1-34]), als Peptidfragment muss das Präparat parenteral verabreicht werden. Das Teriparatid wirkt hauptsächlich über eine Stimulation der osteoblastären Knochenneubildung. Der Einsatz von Teriparatid (20 $\mu \mathrm{g}$ als subkutane tägliche Dosis) ist zum jetzigen Zeitpunkt limitiert für die Behandlung postmenopausaler Frauen (und Männern) mit manifester Osteoporose und hohem Frakturrisiko (prävalente Frakturen, T-Score <-3.5 SD), insbesondere bei Patienten mit ungenügendem bzw. fehlendem Ansprechen auf herkömmliche antiresorptive Therapien (Knochenmassenverlust oder neue Frakturen unter Bisphosphonaten) [46].

Bei postmenopausalen Frauen mit Osteoporose wurde nach einer 19-monatigen Behandlung mit Teriparatid eine signifikante Reduktion der Inzidenz von vertebralen (65\%) und nicht vertebralen Frakturen (53\%) gezeigt [47]. Im praktischen Alltag werden die meisten Patienten, welche sich für eine Behandlung mit Teriparatid qualifizieren, bereits unter einer antiresorptiven Therapie stehen. Im Gegensatz zur Vorbehandlung mit Östrogenen oder Raloxifen scheint eine vorgängige Alendronat-Therapie den Anstieg der Knochendichte an der LWS insbesondere in den ersten 6 Behand- lungsmonaten zu hemmen [48]. Dieser inhibitorische Effekt ist auf die im Gegensatz zu Raloxifen oder Östrogenen ausgeprägtere antiresorptive Wirkung der Bisphosphonate zurückzuführen. Ob diese verminderte Parathormonwirksamkeit auch einen Einfluss auf das Frakturrisiko hat, ist unklar. Die Schlussfolgerungen für den klinischen Alltag sind, dass eine Therapie mit einem SERM oder mit Östrogenen zusammen mit der Teriparatid-Therapie weitergeführt werden kann, die Bisphosphonat-Therapie aber bei Beginn der Teriparatide-Behandlung abgesetzt werden sollte. Evt. ist sogar sinnvoll eine Bisphosphonat-Therapie ca. 6 Monate vor Beginn der Teriparatid-Therapie abzusetzen.

Bei postmenopausalen Frauen mit Osteoporose scheint der knochenanabole Effekt von Teriparatid auf die Behandlungsdauer beschränkt zu sein und nimmt nach dessen Absetzen kontinuierlich ab während die Frakturrisikoreduktion fortbesteht [49, 50]. Dieser Knochenmassenverlust kann sowohl mit einer anschliessenden Östrogentherapie als auch mit einer Alendronatbehandlung aufgehalten werden. Bis dahin scheint es aufgrund der bestehenden Datenlage sinnvoll, im Anschluss an eine 12- bis18-monatige Therapie mit Teriparatid eine antiresorptive Behandlung einzuleiten.

\section{Strontium-Ranelat}

Der molekulare Wirkungsmechanismus von Strontium-Ranelat ist noch ungeklärt. In-vitro- und In-vivo-Studien haben gezeigt, dass Strontium-Ranelat sowohl die Kollagensynthese osteoblastärer Zellen stimuliert als auch die Resorptionsfähigkeit von Osteoklasten hemmt. Entsprechend stellt StrontiumRanelat die erste Substanz dar, welche über einen direkten dualen Effekt auf den Knochenanbau und den Knochenabbau die Knochenmasse günstig beeinflusst. Die fraktursenkende Wirkung von Strontium-Ranelat wurde in zwei randomisierten kontrollierten Studien untersucht. Dabei konnte bei älteren postmenopausalen Frauen mit manifester Osteoporose nach einer Beobachtungszeit von 3 Jahren verglichen zu Placebo eine signifikante Reduktion vertebraler (41\%) [51] und nicht-verte- braler Frakturen (16\%) [52] dokumentiert werden.

\section{Weitere Massnahmen}

$\mathrm{Zu}$ den oben erwähnten medikentösen Massnahmen ergänzen sich v.a. bei älteren Frauen allgemeine präventive Massnamen zur Reduktion des Sturzrisikos (Überprüfung des häuslichen Milieus, Verschreibung von Gehhilfen, Geh- und Sturztraining, Visuskorrektur, Überprüfung des Gebrauchs und der Indikation von Psychopharmaka), der Einsatz von Hüftprotektoren. Der günstige prophylaktische Effekt der Mehrzahl dieser nicht-medikamentösen Massnahmen ist auf die Dauer der Intervention beschränkt und lässt nach deren Sistieren rasch in ihrer Wirkung wieder nach. Es ist allerdings nicht auszuschliessen, dass ein Teil dieser Massnahmen über eine Positivierung der Knochenbilanz eine nachhaltige Wirkung auf die Knochengesundheit ausübt.

\section{Zusammenfassung}

Die klinische Bedeutung der Osteoporose ergibt sich aus ihren Komplikationen, den osteoporotischen Frakturen. Basierend auf epidemiologischen Studien kann erwartet werden, dass jede 2. bis 3. postmenopausale Frau im Laufe des Lebens eine osteoporotische Fraktur erleiden wird.

Die Indikation zur Behandlung der Osteoporose stützt sich auf das individuelle Frakturrisiko, das durch die integrale Beurteilung verschiedener Risikofaktoren (z.B. Alter, Knochendichte, Knochenumbaurate, Vorliegen nichttraumatischer Frakturen, Körpergewicht, Kalzium- und Vitamin-D-Versorgung) ermittelt wird.

Die Kombination einzelner Risikofaktoren zur Voraussage des Frakturrisikos ist der alleinigen Bestimmung der Knochendichte überlegen. Die Entscheidung, welcher Art die Behandlung (präventiv, therapeutisch) sein soll, ergibt sich also erst im Kontext aller Faktoren, die zu einer Erhöhung des Frakturrisikos führen und ist individuell zu fällen. Die Indikation zur Intervention sollte sich nicht nur auf einen einzelnen Knochendichtewert 
abstützen. Bei der Interpretation der Densitometrieresultate muss somit unterschieden werden zwischen der diagnostischen Schwelle (WHO-Definition der Osteoporose: T-Score $\leq-2.5$ ) und der Interventionsschwelle.

In den letzten 15 Jahren sind mehrere grosse placebokontrollierte Doppelblindstudien mit dem klinischen Endpunkt neuer vertebraler und nichtvertebraler Frakturen v.a. bei postmenopausalen Frauen mit Osteoporose publiziert worden. Dabei konnte für verschiedene medikamentöse Wirkprinzipien (Bisphosphonate, Raloxifen, Teriparatid) eine signifikante Reduktion der Inzidenz vertebraler Frakturen zwischen $30 \%$ und $65 \%$ dokumentiert werden. Eine Reduktion nichtvertebraler Frakturen wurde insbesondere für die Bisphosphonate Alendronat und Risedronat als auch für Teriparatid gezeigt. Im klinischen Alltag richtet sich die Wahl eines spezifischen Therapeutikums nach dem individuellem Frakturrisiko und sollte unter Abwägung skeletaler und nichtskeletaler Medikamentenwirkungen bzw. -nebenwirkungen erfolgen.

\section{Literatur}

1. Roy DK et al. Determinants of incident vertebral fracture in men and women: results from the european prospective osteoporosis study (EPOS). Osteoporos prospective osteop
Int 2003;14:19-26

2. Melton LJ, III. Epidemiology worldwide. Endocrinol Metab Clin North Am 2003;32:1-13

3. Adachi JD, loannidis G, Pickard L, Berger C, Prior JC, Joseph $L$ et al. The association between osteoporotic fractures and health-related quality of life as measured by the Health Utilities Index in the Canadian Multicentre Osteoporosis Study (CaMos). Osteoporos Int. 2003;14:895-904.

4. Nevitt MC, Ettinger B, Black DM, Stone K, Jamal $\mathrm{SA}$, Ensrud $\mathrm{K}$ et al. The association of radiographically detected vertebral fractures with back pain and function: a prospective study. Ann Int Med. 1998; function: a prosp
128:793-800.

5. Cauley JA, Thompson DE, Ensrud KC, Scott JC, Black D. Risk of mortality following clinical fractures. Osteoporos Int. 2000;11:556-61.

6. Johnell O, Kanis JA, Oden A, Sernbo I, RedlundJohnell I, Petterson C et al. Mortality after osteoporotic fractures. Osteoporos Int. 2004;15:38-42.

7. Brown JP, Josse RG. 2002 clinical practice guidelines for the diagnosis and managment of osteo-

8. Seeman E. Invited Review: Pathogenesis of osteoporosis. J Appl Physiol. 2003;95:2142-51.

9. Rosen CJ. Pathogenesis of osteoporosis. Best Pract Res. 2000;14:181-93.

10. Meier C, Handelsman DJ, Seibel MJ. Endocrine regulation of bone turnover in men. Clin Endocrinol. 2005;62:in print.

11. Scheidt-Nave C, Baum E, Dören M, Hadji P, Minne H. DVO-Leitlinien Osteoporose bei postmenopausaIen Frauen. Osteologie. 2003; 12:63-91.

12. Kanis JA. Diagnosis and osteoporosis and assessment of fracture risk. Lancet. 2002; 359:1929-36.

13. Kanis JA, Johnell O, Oden A, De Laet C, Oglesby A, Jonsson B. Intervention thresholds for osteoporosis. Bone. 2002:31:26-31.

14. Dachverband Osteologie. Leitlinie 2006 des DVO zur
Prophylaxe, Diagnostik und Therapie der Osteoporose bei Frauen ab der Menopause und bei Männern ab dem 60.Lebensjahr. www dvo-osteologie org. 2005.

15. de Laet CE, van der KM, Hofman A, Pols HA. Osteoporosis in men and women: a story about bone mineral density thresholds and hip fracture risk. J Bone Miner Res. 2002; 17:2231-36.

16. Kanis JA, Johnell O, Oden A, De Laet C, Mellstrom D. Diagnosis of osteoporosis and fracture threshold in men. Calcif Tissue Int. 2001:69:218-21.

17. Mohr A, Barkmann R, Mohr C, Romer FW, Schmidt C, Heller $\mathrm{M}$ et al. [Quantitative ultrasound for the diagnosis of osteoporosis]. Fortschr Röntgenstr. 2004;176:610-617.

18. Gluer CC, Eastell R, Reid DM, Felsenberg D, Roux C, Barkmann R et al. Association of Five Quantitative Ultrasound Devices and Bone Densitometry With Osteoporotic Vertebral Fractures in a PopulationBased Sample: The OPUS Study. J Bone Miner Res. 2004; 19:782-93.

19. Hans D, Dargent-Molina P, Schott AM, Sebert JL, Cormier C, Kotzki PO et al. Ultrasonographic heel measurements to predict hip fracture in elderly women: the EPIDOS prospective study. The Lancet. 1996:348: 511-14.

20. Krieg MA, Comuz J, Ruffieux C, Burckhardt P. [Role of bone ultrasound in predicting hip fracture risk in of bone ultrasound in predicting hip fracture risk in women 70 years or older: results of the SEMOF
study and comparison with literature data]. Rev Med Suisse Romande. 2004;124:59-62.

21. Hans D, Hartl F, Krieg MA. Device specific weighted T-score for two quantitative ultrasounds: operational proposition for the management of osteoporosis for 65 years and older women in Switzerland. Osteoporos Int. 2003:14:251-58.

22. Eastell R, Barton I, Hannon RA, Chines A, Garnero P, Delmas P. Relationship of early changes in bone resorption to the reduction in fracture risk with risedronate. J Bone Miner Res. 2003;18:1051-56.

23. Garnero P, Delmas PD. Contribution of bone mineral density and bone turnover markers to the estimation of risk of osteoporotic fracture in postmenopausal of risk of osteoporotic fracture in postmenopausal 50-63.

24. Johnell O, Odén A, De Laet C, Delmas PD, Kanis JA. Biochemical Indices of Bone Turnover and the Assessment of Fracture Probability. Osteoporos Int. 2002;13:523-26.

25. Alekel DL, Germain AS, Peterson CT, Hanson KB, Stewart JW, Toda T. Isoflavone-rich soy protein isolate attenuates bone loss in the lumbar spine of perimenopausal women. Am J Clin Nutr. 2000;72: 844-52

26. Kanis JA, Borgstrom F, De Laet C, Johansson $H$, Johnell O, Jonsson B et al. Assessment of fracture risk. Osteoporos Int. 2005;16: 581-89.

27. Delmas PD, Genant HK, Crans GG, Stock JL, Wong $M$, Siris $E$ et al. Severity of prevalent vertebral fractures and the risk of subsequent vertebral and nonvertebral fractures: results from the MORE trial. Bone. 2003;33:522-32

28. Johnell O, Kanis JA, Odén A, Sernbo I, RedlundJohnell I, Petterson $\mathrm{C}$ et al. Fracture risk following an osteoporotic fracture. Osteoporos Int. 2004;15: 175-79.

29. Kanis JA, Johnell O, De Laet C, Johansson H, Oden A, Delmas $P$ et al. A meta-analysis of previous fracture and subsequent fracture risk. Bone. 2004;35: 375-82

30. Incidence of vertebral fracture in europe: results from the European Prospective Osteoporosis Study (EPOS). J Bone Miner Res. 2002;17:716-24.

31. Lecouvet FE, Malghem J, Maldague BE, Vande Berg BC. MR imaging of epiphyseal lesions of the knee: current concepts, challenges, and controversies. Radiol Clin North Am. 2005;43:655-viii.

32. Pientka L, Baum E, Kruse H-P, Pfeilschifter J, Ringe JD. DVO-Leitlinie Osteoporose des älteren Menschen. Osteologie. 2003;12: 93-117.

33. Chapuy MC, Arlot ME, Duboeuf F, Brun J, Crouzet B, Arnaud $S$ et al. Vitamin D3 and calcium to prevent hip fractures in elderly women. $N$ Engl $\mathrm{J}$ Med. 1992;327:1637-42.

34. Dawson-Hughes B, Harris SS, Krall EA, Dallal GE. Effect of calcium and vitamin D supplementation on bone density in men and women 65 years of age bone density in men and women 65 years
and older. N Engl J Med. 1997;337:670-676.

35. Porthouse J, Cockayne S, King C, Saxon L, Steele E, Aspray $T$ et al. Randomised controlled trial of calcium and supplementation with cholecalciferol (vitamin D3) for prevention of fractures in primary care. Br Med J. 2005;330:1003

35A. Jackson RD, LaCroix AZ, Gass M, Wallace RB, Robbins J, Lewis CE et al. Calcium plus vitamin D sup- plementation and the risk of fractures. N.Engl.J Med. 2006;354:669-83

35B. Finkelstein JS. Calcium plus vitamin D for postmenopausal women-bone appetit? N.Engl.J Med. 2006;354:750-52

36. Hulley S, Grady D, Bush T. Randomised trial of estrogen plus progestin for secondary prevention of coronary heart disease in post-menopausal women. JAMA. 1998;280:605-13.

37. Writing Group for the WHI Investigators. Risk and benefits of estrogen plus progestin in healthy post-
menopausal women. JAMA. 2002;288:321-33.

38. Anderson GL, Limacher M, Assaf AR, Bassford T, Beresford SA, Black $\mathrm{H}$ et al. Effects of conjugated equine estrogen in postmenopausal women with hysterectomy: the Women's Health Initiative randomized controlled trial. JAMA. 2004:291:1701-12.

39. Ettinger $B$, Black DM, Mitlak BH, Knickerbocker RK Nickelsen T, Genant HK et al. Reduction of vertebral fracture risk in postmenopausal women with osteoporosis treated with raloxifene (results from a 3-yea randomized clinical trial). JAMA. 1999;282:637-45.

40. Black DM, Cummings StR, Karpf DB, Cauley JA Thompson DE, Nevitt MC et al. Randomised trial of effect of alendronate on risk of fracture in women with existing vertebral fractures. The Lancet. 1996;348:1535-41.

41. Cummings SR, Black DM, Thompson DE, Applegate WB, Barett-Connor E, Musliner TA et al. Effect of alendronate on risk of fracture in women with low bone density but without vertebral fractures. JAMA 1998;280:2077-82

42. Bone HG, Hosking D, Devogelaer J-P, Tucci JR, Emkey RD, Tonino RP et al. Ten years' experience with alendronate for osteoporosis in post menopausal women. The New England Journal of Medicine. 2004;350:1189-99.

43. Harris ST, Watts NB, Genant HK, McKeever CD, Hangartner $\mathrm{T}$, Keller $\mathrm{M}$ et al. Effects of Risedronate treatment on vertebral and nonvertebral fractures in women with postmenopausal osteoporosis. JAMA. 1999;282: 1344-52

44. McClung MR, Geusens P, Miller PD, Zippel H, Bensen WG, Roux $C$ et al. Effect of risedronate on the risk of hip fracture in elderly women. N Engl Med. 2001;344:333-40.

45. Chesnut $\mathrm{CH}$, Skag A, Christiansen $\mathrm{C}$, Recker $\mathrm{R}$ Stakkestad JA, Hoiseth A et al. Effects of oral ibandronate administered daily or intermittently on fracture risk in postmenopausal osteoporosis. J Bone Miner Res. 2004;19: 1241-49.

46. Hodsman AB, Bauer DC, Dempster D, Dian L, Hanley DA, Harris ST et al. Parathyroid hormone and teriparatide for the treatment of osteoporosis: a review of the evidence and suggested guidelines for its use. Endocr Rev. 2005.

47. Neer RM, Arnaud CD, Zanchetta JR, Prince R, Gaich GA, Reginster J-Y et al. Effect of parathyroid hormone (1-34) on fractures and bone mineral density in postmenopausal women with osteoporosis. New Engl J Med. 2001;344:1434-41.

48. Ettinger B, San MJ, Crans G, Pavo I. Differentia Effects of Teriparatide on BMD After Treatment
With Raloxifene or Alendronate. J Bone Miner Res. 2004;19:745-51.

49. Lindsay R, Scheele WH, Neer R, Pohl G, Adami S Mautalen $\mathrm{C}$ et al. Sustained vertebral fracture risk reduction after withdrawal of teriparatide in postmenopausal women with osteoporosis. Arch Intern Med. 2004;164: 2024-30.

50. Kaufman JM, Orwoll E, Goemaere S, San MJ, Hossain A, Dalsky GP et al. Teriparatide effects on vertebral fractures and bone mineral density in men with osteoporosis: treatment and discontinuation of therapy. Osteoporos Int. 2005;16:510-516.

51. Meunier PJ, Roux C, Seeman E, Ortolani S, Badurski JE, Spector TD et al. The effects of strontium ranelate on the risk of vertebral fracture in women with postmenopausal osteoporosis. N Engl J Med. 2004;350: 459-68.

52. Reginster JY, Seeman E, De Vernejoul MC, Adami S, Compston J, Phenekos $\mathrm{C}$ et al. Strontium ranelate reduces the risk of nonvertebral fractures in postmenopausal women: TROPOS study. J Clin Endocrinol Metab. 2005.

\section{Korrespondenzadresse:}

PD Dr.med. Marius Kraenzlin

Missionsstrasse 24, CH-4055 Basel

e-mail: marius.kraenzlin@unibas.ch 